\title{
BIBECHANA
}

A Multidisciplinary Journal of Science, Technology and Mathematics ISSN 2091-0762 (Print), 2382-5340 (0nline)

Journal homepage: http://nepjol.info/index.php/BIBECHANA

Publisher: Research Council of Science and Technology, Biratnagar, Nepal

\section{Antibiotic Susceptibility Pattern of Biofilm forming Uropathogenic Escherichia coli Isolated from UTI infected Patients of Koshi Zonal Hospital in Biratnagar, Nepal}

\author{
S. Chaudhary ${ }^{1}$, B. Khatiwada ${ }^{1}$, N. K. Chaudhary ${ }^{2 *}$, \\ ${ }^{1}$ Department of Microbiology, Mahendra Morang Adarsh Multiple Campus, Biratnagar, Nepal \\ ${ }^{2}$ Department of Chemistry, Mahendra Morang Adarsh Multiple Campus, Biratnagar, Nepal \\ Tribhuvan University \\ "Email: chem_narendra@yahoo.com
}

Article history: Received 3 September, 2018; Accepted 13 September, 2018

DOI: http://dx.doi.org/10.3126/bibechana.v16i0.19192

This work is licensed under the Creative Commons CC BY-NC License.

https://creativecommons.org/licenses/by-nc/4.0/

\begin{abstract}
Objectives: To investigate the prevalence and antibiotic resistance pattern of biofilm-forming Uropathogenic Escherichia coli (UPEC) from urine samples isolated from UTI infected patients of Koshi zonal hospital, Biratnagar.

Methods: A total of 51 urine samples from urinary tract infected patients were collected from Koshi zonal hospital, Biratnagar in the period of July to August 2017. Following the isolation and identification of biofilm-forming uropathogenic Escherichia coli, antibiotic susceptibility test was performed by a modified Kirby-Bauer disc diffusion technique. The biofilm detection was done by Congo red agar method.

Results: In the present study, $45 \%$ of the urine samples showed a predominant growth of $E$. coli, among which $70 \%$ of isolates exhibited positive biofilm formation. Biofilm forming isolates revealed $100 \%, 87.5 \%, 75 \%, 63 \%$ and $12.5 \%$ resistant to erythromycin, amoxicillin, cefotaxime, levofloxacin, and nitrofurantoin respectively. Approximately $87.5 \%$ of biofilm-forming isolates were found multidrug resistant.

Conclusion: The study revealed the major issue of UTI by E. coli which may be due to poor sanitation, not the proper cleanliness of genitals and unsafe sexual intercourse. Nitrofurantoin and levofloxacin were examined the most effective antibiotics for UPEC.
\end{abstract}

Keywords: Biofilm; urinary tract infection; antibiotic susceptibility; E. coli; multidrug resistance.

\section{Introduction}

Urinary tract infections have become a major public health concern in the medical science and represent one of the most common infectious disease ranking next to upper respiratory tract infection. They contribute to the nosocomial infection in many hospitals and account for approximately $35 \%$ of 
all hospital-acquired infections [1]. Unhygienic sexual intercourse and not washing the genitals properly have made the urinary tract infection prevalence in the urban areas of Nepal. Infection by uropathogenic Escherichia coli has made a worldwide problem mostly affecting the women in both developed and underdeveloped countries [2]. It is the most important pathogen associated with UTI and the primary etiological agent among both outpatients and inpatients. Common pathogens that have been implicated in UTIs are primarily gram-negative organisms with $E$. coli having a more prevalence than others including K. pneumoniae, Enterobacter spp., P. mirabilis, $P$. aeruginosa and Citrobacter spp. The organism is therefore of clinical importance and can be isolated from various clinical specimens $[3,4]$. It is one of the organisms most frequently isolated from urine. In addition to urinary tract infection, E. coli is the most frequent pathogen associated with intra-abdominal infection. The biofilm forming UPEC has made much more difficulties in the eradication and elimination of the urinary tract infection [5]. The resistance of bacteria to antimicrobials may pose a serious threat in both developing and developed countries. Patients with infections by resistant organisms are at an increased risk of treatment failure [6]. Bacterial biofilms generally provide a protective environment for their constituent bacteria, shielding them from antibiotics and host immune effects, and it seems quite likely that biofilms in the bladder may account for the recalcitrance of UPEC to treatment and clearance from the urinary tract $[7,8]$.

In the present investigation, we aimed to detect the biofilm forming uropathogenic E. coli isolated from UTIs infected patients of Koshi zonal hospital, Biratnagar and evaluated their antibiotic susceptibility pattern. We have also examined the isolates that showed drug resistance.

\section{Materials and Methods}

\subsection{Sample collection and processing}

Urine samples were collected from Koshi zonal hospital, Biratnagar from July to August 2017. A total of 51 freshly voided midstream urine samples $(10-20 \mathrm{ml})$ were collected aseptically from UTI suspected patients in the wide-mouthed sterile plastic vial. The collected urine samples were kept in the icebox at $4{ }^{\circ} \mathrm{C}$ during transportation and analyzed in Microbiology Laboratory of Mahendra Morang Adarsh Multiple Campus, Biratnagar, on the same day immediately after their delivery within 6 hours of collection.

\subsection{Isolation and identification of E.coli from urine samples}

$0.1 \mu \mathrm{L}$ of each urine sample received from the patients were spread on Eosine Methylene Blue (EMB) agar by spread plate method for the growth of bacteria and the culture plates were incubated in an inverted position at $37^{\circ} \mathrm{C}$ under the aerobic condition for 24-48 hours [9]. After growth of organisms, the pink greenish metallic sheen was inoculated on nutrient agar medium for each discrete colony presented in the specimen to obtain well- isolated pure colonies incubated at $37{ }^{\circ} \mathrm{C}$ for 24 hours. Isolated colonies were subjected to biochemical tests such as catalase, oxidase, MR, VP, urease, citrate, indole, TSI to identify the E. coli based on the Bergey's manual of determination of bacteriology [10]. The Figure (4 a-d) presents isolation and identification of biofilm-forming UPEC and AST pattern performed by E. coli.

\subsection{Identification of biofilm-forming UPEC}

The well identified E. coli were subjected to biofilm formation by Congo red agar method [11]. The isolated colony was streaked on the Congo red agar (CRA) and incubated for 24 hours at $37^{\circ} \mathrm{C}$. After the incubation, the biofilm forming UPEC showed black colonies with dry crystalline consistency. 
Red or pink colonies are considered as nonbiofilm producers and the pink colony with black centered is considered as moderate biofilm producer [12].

\subsection{AST pattern of biofilm-forming UPEC}

Antibiotic susceptibility test of the biofilm forming UPEC towards various antimicrobial discs such as Nitrofurantoin (300 mcg/disc), Levofloxacin $(5 \mathrm{mcg} / \mathrm{disc})$, Cefotaxime (30 mcg/disc), Erythromycin $(15 \mathrm{mcg} / \mathrm{disc})$ and Amoxicillin $(10 \mathrm{mcg} / \mathrm{disc})$ was done by modified Kirby-Bauer disk diffusion method on Mueller Hilton agar [13]. Organisms were classified as sensitive or resistant to an antibiotic according to the diameter of the zone of inhibition surrounding each antibiotic disc based on the Clinical and Laboratory Standards Institute guidelines [14]. Based on the sensitive pattern, isolates resistant to three or more antibiotics were considered as multidrug-resistant bacteria.

\section{Results}

Fifty-one urine samples of urinary tract infected patients were processed, among which 15 (29.41\%) samples gave significant growth, 8 (15.69\%) samples gave mixed growth and 7 (13.73\%) samples (Figure 1) gave non- significant growth while remaining samples showed no growth. E. coli was found to be the most predominant isolate in $23(45.09 \%)$ samples. The distribution of E. coli was found to be the most frequent in age groups 13-55 years in both sexes i.e. 3 in male and 20 in the female. The sex wise distribution (Figure 2) showed that females $(86.97 \%)$ were more susceptible than males (13.04\%). Out of 51 samples, 23 samples showed the growth of $E$. coli which were subjected to biofilm productions by Congo red agar method among which $16(70 \%)$ isolates showed the formation of biofilms [15]. All the biofilm producing UPEC were subjected to antibiotic susceptibility test $[16,17]$ which showed maximum resistant towards erythromycin $(100 \%)$ followed by amoxicillin (87.5\%), cefotaxime (75\%), levofloxacin (63\%) and nitrofurantoin $(12.5 \%)$. Thus, maximum susceptibility was shown by nitrofurantoin $(87.5 \%)$ and the least effective drug was erythromycin $(0 \%)$. The bar graph analysis for antibiotic susceptibility pattern of biofilm-forming UPEC is given in Figure 3 and the growth inhibition data is reported in Table 1.

Table 1: Antimicrobial susceptibility pattern of biofilm-forming UPEC (\%).

\begin{tabular}{lll}
\hline Antibiotics & Sensitive & Resistant \\
\hline Amoxicillin & 12.5 & 87.5 \\
Cefotaxime & 25 & 75 \\
Erythromycin & 0 & 100 \\
Levofloxacin & 37 & 63 \\
Nitrofurantoin & 87.5 & 12.5 \\
\hline
\end{tabular}

Multidrug resistance is defined as the resistance to more than three antibiotics or more. Taking resistant to two or more classes of antibiotics as MDR, it was detected that $14(87.5 \%)$ isolates were resistant to more than three antibiotics. The MDR data for biofilm-forming UPEC is reported in Table 2 .

\section{Discussion}

The antibiotic-resistant pathogens have resulted in an increment of health care cost worldwide. Not only the resistant to antibiotics, the biofilm production by an organism has made it more difficult for curing. The availability of antibiotics seems to be inadequate in the context of increasing resistant problems. Antibiotic resistance is a common phenomenon in the developing country where antibiotics are available freely without the prescription $[18,19]$. The antibiotic susceptibility pattern varies 
among the hospitals and nations from one country to another and also depends upon the variation of geographical regions. Therefore therapeutic success against UPEC depends upon the local susceptibility profiles [20]. This study was done to screen the resistance pattern of antibiotics among the biofilm producing uropathogenic Escherichia coli.

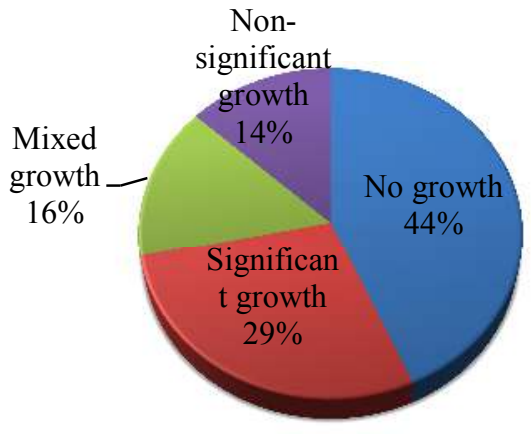

Fig. 1: Pie-chart distribution of bacteria.

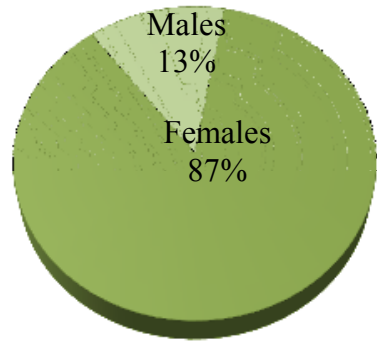

Fig. 2: Pie-chart distribution of E. coli according to sex.

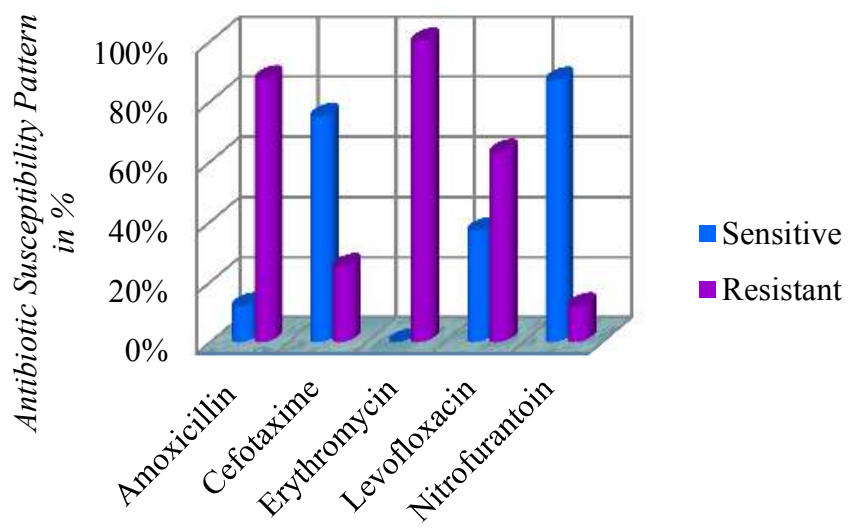

Fig. 3: Antibiotic susceptibility bar graph of biofilm-forming UPEC.

Table 2: Multidrug-resistant pattern of biofilm-forming UPEC

\begin{tabular}{|c|c|c|c|c|c|c|c|}
\hline \multirow{2}{*}{$\begin{array}{l}\text { Bacterial } \\
\text { Isolates }\end{array}$} & \multirow{2}{*}{$\begin{array}{l}\text { No. of } \\
\text { biofilm } \\
\text { isolates }\end{array}$} & \multicolumn{4}{|c|}{ MDR Strain } & \multirow{2}{*}{\multicolumn{2}{|c|}{ Total }} \\
\hline & & 0 drug & 1 drug & 2 drug & $\begin{array}{l}2 \\
\text { drug }\end{array}$ & & \\
\hline E. coli & 16 & 0 & 2 & 0 & 14 & 14 & $87.5 \%$ \\
\hline
\end{tabular}




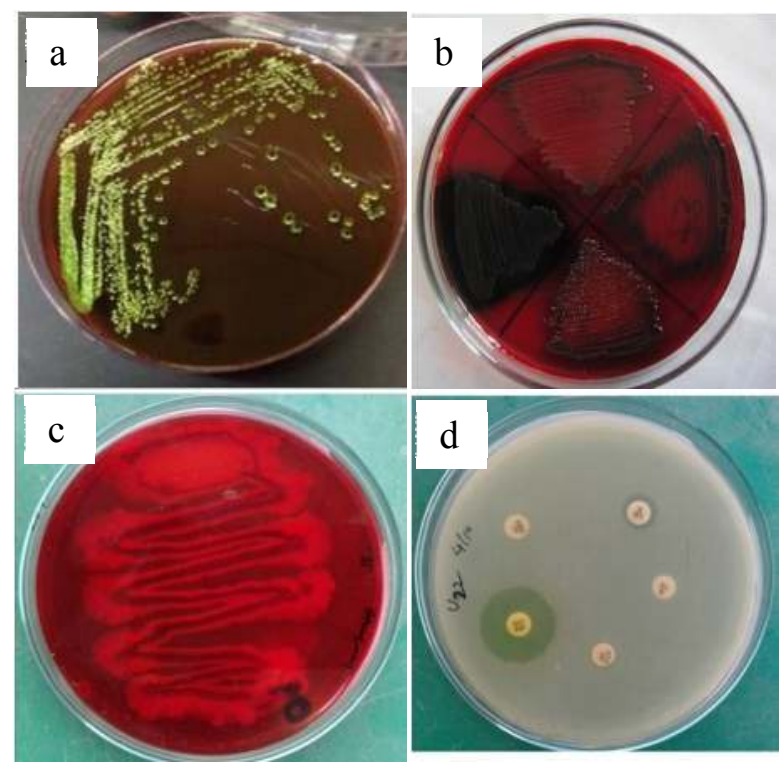

Fig. 4: (a) E. coli on eosin methylene blue (EMB) agar media. (b) Biofilm forming UPEC (high growth).

(c) Biofilm forming UPEC (moderate growth) (d) AST of E. coli performed on MHA.

Biofilm formation is closely related with the resistance of UPEC towards the beta-lactam antibiotics; amoxicillin and cefotaxime, and the macrolides; erythromycin. They are hardly effective antimicrobial drugs and also increase the chronicity of urinary tract infection. The high resistance of biofilms towards $\beta$-lactam antibiotics and macrolides may be due to restricted penetration of antimicrobial agents into biofilms, nutrients limitation, gene expression in the stress response and further, due to the more space in the biofilm rather than that of planktonic cells. The genetic exchange takes place between the resistance and nonresistance strains and finally increase the emergence of multidrug-resistant strains of UPEC [21-23]. Antibiotics resistance in biofilm forming UPEC is further aided by the irrational exposure of several antibiotics without precise prescription which is very common in the developing country like Nepal.

Our study was conducted among outpatients suspected of UTI, attending Koshi zonal hospital of Biratnagar. In this research, the analytical data presented in the result section in response to the growth of biofilm-forming E. coli are in good agreement with other investigators. In our study, 23 $(45.09 \%)$ E. coli isolated from 8 significant and 7 mixed growth urine samples was found the commonest organism isolated. Almost similar results were observed in the literature [24, 25] and $E$. coli is considered as the predominant pathogen for biofilm-forming UPEC.

Uropathogenic $E$. coli was isolated most commonly from female patients compared to male patients. The study showed that $87 \%$ female were susceptible to UTI in comparison to male $13 \%$. The increased rate of UTI in the female is due to anatomic factors, urodynamic disturbance, and hormonal changes. Biofilm producing UPEC played a significant role in the antibiotic resistance and incurable stages leading to high morbidity and mortality. Among 23 E. coli isolates, $16(70 \%)$ isolates showed biofilm formation dividing it into high, moderate and low biofilm formation. The results are in good agreement with the literature [26-29]. 
The investigation revealed that the biofilm forming UPEC were mostly resistant to erythromycin $(100 \%)$ followed by amoxicillin (86\%) which resembles other studies [7,26,27,29]. On the basis of our findings, antibiotic such as erythromycin and amoxicillin should be no longer recommended for initial therapeutics for urinary tract infections caused by biofilm-forming UPEC.

Our study also showed that only $2(12.5 \%)$ biofilm forming UPEC were resistant to Nitrofurantoin which is similar to the findings of other researchers. In the context of Nepal, Nitrofurantoin is the best antibiotic prescribed for the case of UTI and biofilm forming UPEC [7]. However resistant to Quinolones and Fluoroquinolones has been increasing day by day. The major consequences being the use of the antibiotics at the poultry and the resistant strain being transmitted from animal to the human beings [30, 31].

\section{Conclusion}

In Nepalese context, the biofilm forming UPEC and the MDR strains of E. coli, being more prevalence of urinary tract infection, are an alarming signal of health hazard which will be very difficult to control in the future. In conclusion, the major issue of UTI infection is due to E. coli which were about $45.09 \%$. The poor sanitary condition, lack of health education, careless in the cleanliness of genitals and unsafe sexual intercourse are playing the leading role in UTI infection. According to the antibiotic sensitivity test, nitrofurantoin and levofloxacin are the most effective antibiotics for the treatment of UTI. Erythromycin and amoxicillin are not active against the UTI infections due to drug resistance and these should not be used. The Biofilm forming capability of the UPEC poses a great challenge to clinicians and microbiologist and therefore, the major focus should be on drug resistance, so that we can minimize the mortality and morbidity.

\section{Declaration of interest}

The authors declare no conflicts of interest. The content and writing of the paper are all under the author's responsibility.

\section{References}

[1] A. L. Flores-Mireles, J. .N. Walker, M. Caparon, S.J. Hultgren, Urinary tract infections: epidemiology, mechanisms of infection and treatment options, Nature Rev. Microbiol. 13 (2015) 269-284. doi:10.1038/nrmicro3432.

[2] D.J. Schwartz, M.S. Conover, T.J. Hannan, S.J. Hultgren, Uropathogenic Escherichia coli superinfection enhances the severity of mouse bladder infection, PLoS Pathog. 11 (2015) 1-12. doi:10.1371/journal.ppat.1004599.

[3] S.M. Jacobsen, D.J. Stickler, H.L.T. Mobley, M.E. Shirtliff, Complicated catheter-associated urinary tract infections due to Escherichia coli and Proteus mirabilis, Clin. Microbiol. Rev. 21 (2008) 26-59. doi:10.1128/CMR.00019-07.

[4] S. Rath, R.N. Padhy, Surveillance of acute community acquired urinary tract bacterial infections, J Acute Dis. 4 (2015) 186-195. doi:10.1016/j.joad.2015.06.001.

[5] L. Ferrières, V. Hancock, P. Klemm, Biofilm exclusion of uropathogenic bacteria by selected asymptomatic bacteriuria Escherichia coli strains, Microbiol. 153(2007)1711-1719. doi:10.1099/mic.0.2006/004721-0.

[6] E. Toubes, K. Singh, D. Yin, R. Lyu, N. Glick, L. Russell, S. Mohapatra, N. Saghal, R.A. Weinstein, G. Trenholme, Risk factors for antibiotic-resistant infection and treatment outcomes among hospitalized patients transferred from long-term care facilities: does antimicrobial choice make a difference?, Clin. Infect. Dis. 36 (2003) 724-730. doi:10.1086/368081.

[7] S. Neupane, N.D. Pant, S. Khatiwada, R. Chaudhary, M.R. Banjara, Correlation between biofilm 
formation and resistance toward different commonly used antibiotics along with extended spectrum beta lactamase production in uropathogenic Escherichia coli isolated from the patients suspected of urinary tract infections visit, Antimicrob. Resist. Infect. Control. 5 (2016) 1-5. doi:10.1186/s13756-016-0104-9.

[8] G.G. Anderson, S.M. Martin, S.J. Hultgren, Host subversion by formation of intracellular bacterial communities in the urinary tract, Microbes and Infect. 6 (2004) 1094-1101. doi:10.1016/j.micinf.2004.05.023.

[9] W.L. Tsai, C.E. Miller, E.R. Richter, Determination of the sensitivity of a rapid Escherichia coli O157:H7 assay for testing 375-gram composite samples, Appl. Environ. Microbiol. 66 (2000) 41494151. doi:10.1128/AEM.66.9.4149-4151.2000.

[10] Bergey's Manual ${ }^{\circledR}$ of Systematic Bacteriology, Springer US. 2 (2005). doi:10.1007/0-387-29298-5.

[11] A. Hassan, J. Usman, F. Kaleem, M. Omair, A. Khalid, M. Iqbal, Evaluation of different detection methods of biofilm formation in the clinical isolates, Braz. J. Infect. Dis. 15 (2011) 305-311. doi:10.1016/S1413-8670(11)70197-0.

[12] P. Subramanian, N. Shanmugam, U. Sivaraman, S. Kumar, S. Selvaraj, Antibiotic resistance pattern of biofilm-forming uropathogens isolated from catheterized patients in Pondicherry, India, Australas. Med. J. 5 (2012) 344-348. doi: 10.4066/AMJ.2012.1193

[13] A.W. Bauer, W.M. Kirby, J.C. Sherris, M. Turck, Antibiotic susceptibility testing by a standardized single disk method, Am. J. Clin. Pathol. 45 (1966) 493-496. http://www.ncbi.nlm.nih.gov/pubmed/5325707.

[14] Performance standards for antimicrobial disk susceptibility tests; Approved standard-Twelfth edition, Clinical and Laboratory Standards Institute. (2015).

[15] C.P. Bhatt, B. Shrestha, S. Khadka, S. Swar, B. Shah, K. Pun, Etiology of urinary tract infection and drug resistance cases of uropathogenes, J. Kathmandu Med. College. 1 (2012) 114-120. doi.org/10.3126/jkmc.v1i2.8150.

[16] P. Ponnusamy, V. Natarajan, M. Sevanan, In vitro biofilm formation by uropathogenic Escherichia coli and their antimicrobial susceptibility pattern, Asian Pac. J. Trop. Med. 5 (2012) 210-213. doi:10.1016/S1995-7645(12)60026-1.

[17] G.K. Badave, D. Kulkarni, Biofilm producing multidrug resistant acinetobacter baumannii: An emerging challenge, J. Clin. Diagn. Res. 9 (2015) DC08-DC10. doi:10.7860/JCDR/2015/11014.5398.

[18] WHO's first global report on antibiotic resistance reveals serious, worldwide threat to public health, WHO. (2014).

[19] R. E. Istúriz, C. Carbon, Antibiotic use in developing countries, Infect Control Hosp Epidemiol. 21 (2000) 394-397. doi:10.1086/501780.

[20] E. Tajbakhsh, P. Ahmadi, E. Abedpour-Dehkordi, N. Arbab-Soleimani, F. Khamesipour, Biofilm formation, antimicrobial susceptibility, serogroups and virulence genes of uropathogenic E. coli isolated from clinical samples in Iran, Antimicrob. Resist. Infect. Control. 5 (2016). doi:10.1186/s13756-0160109-4.

[21] A.L. Spoering, K. Lewis, Biofilms and planktonic cells of Pseudomonas aeruginosa have similar resistance to killing by antimicrobials, J. Bacteriol. 183 (2001) 6746-6751. doi:10.1128/JB.183.23.6746$\underline{6751.2001 .}$

[22] F. Reffuveille, C. de la Fuente-Nũnez, S. Mansour, R.E. Hancock, A broad-spectrum antibiofilm peptide enhances antibiotic action against bacterial biofilms, Antimicrob. Agents Chemother. 58 (2014) 53635371. doi:10.1128/AAC.03163-14.

[23] J. Haaber, M.T. Cohn, D. Frees, T.J. Andersen, H. Ingmer, Planktonic aggregates of Staphylococcus aureus protect against common antibiotics, PLoS ONE. 7 (2012). doi:10.1371/journal.pone.0041075.

[24] S. Sabir, A. Ahmad Anjum, T. Ijaz, M.A. Ali, M. UR R. Khan, M. Nawaz, Isolation and antibiotic susceptibility of $E$. coli from urinary tract infections in a tertiary care hospital, Pak. J. Med. Sci. 30 (2014) 389-392. doi:10.12669/pjms.302.4289. 
[25] M. Tabasi, M.R. Asadi Karam, M. Habibi, M.S. Yekaninejad, S. Bouzari, Phenotypic assays to determine virulence factors of uropathogenic Escherichia coli (UPEC) isolates and their correlation with antibiotic resistance pattern, Osong Pub. Health Res. Perspect. 6 (2015) 261-268. doi:10.1016/j.phrp.2015.08.002.

[26] P. Poovendran, N. Ramanathan, In vitro study on antibiotic susceptibility pattern of biofilm producing uropathogenic escherichia coli isolates and their molecular characterization, Asian J. Pharmaceut. Clin. Res. 7 (2014) 181-185.

[27] P. Poovendran, N. Vidhya, S. Murugan, Antimicrobial susceptibility pattern of ESBL and non-ESBL producing uropathogenic Escherichia coli ( UPEC ) and their correlation with biofilm formation, Internation. J. Microbiol. Res. 4 (2013) 56-63. doi:10.5829/idosi.ijmr.2013.4.1.71235.

[28] S. Golia, V. Hittinahalli, S.K. Karjigi, K.M. Reddy, Correlation between biofilm formation of uropathogenic Escherichia coli and its antibiotic resistance pattern, J. Evolut. Med. Dent. Sci. 1 (2012) $166-175$.

[29] T. Bajpai, M. Varma, G.S. Bhatambare, M. Pandey, Escherichia coli biofilms : accepting the therapeutic challenges, Internation. J. Health \& Allied Sci. 5 (2016) 204-209. doi:10.4103/2278-344X.194081.

[30] A.B. Lagha, B. Haas, M. Gottschalk, D. Grenier, Antimicrobial potential of bacteriocins in poultry and swine production, Veterinary Res. 48 (2017). doi:10.1186/s13567-017-0425-6.

[31] K. Dhama, V. Verma, P.M. Sawant, R. Tiwari, R.K. Vaid, R.S. Chauhan, Applications of probiotics in poultry: enhancing immunity and beneficial effects on production performances and health - A Review, J. Immunol. Immunopathol. 13 (2011). 